Review Article

\title{
The Effects of Ischemic Preconditioning Supplementation on Endothelial Function: A Systematic Review and Meta-Analysis
}

\author{
Xufang Gu $\mathbb{D},{ }^{1}$ Zhichao Liu $\mathbb{D}^{2},{ }^{2}$ Shengwei Gao, ${ }^{2}$ Li Ma, ${ }^{2}$ Jinhong Chen, ${ }^{2}$ Zhenxing Wang, \\ Anmin Lu, ${ }^{2}$ Zhizhong Wang, ${ }^{2}$ Baohe Wang, ${ }^{1}$ and Yuhong $\mathrm{Li} \mathbb{i D}^{2}$ \\ ${ }^{1}$ The Second Affiliated Hospital of Tianjin University of Traditional Chinese Medicine, Tianjin, China \\ ${ }^{2}$ Tianjin University of Traditional Chinese Medicine, Tianjin, China
}

Correspondence should be addressed to Xufang Gu; 1201930131104@yeah.net and Yuhong Li; liyuhong@tjutcm.edu.cn

Received 25 October 2020; Revised 3 December 2020; Accepted 16 July 2021; Published 26 July 2021

Academic Editor: Abraham Wall-Medrano

Copyright (c) 2021 Xufang Gu et al. This is an open access article distributed under the Creative Commons Attribution License, which permits unrestricted use, distribution, and reproduction in any medium, provided the original work is properly cited.

Objective. Ischemic preconditioning (IPC) has gradually been promoted in clinical practice to lower the risk of cardiovascular surgery and postoperative complications. We investigated the role of IPC on vascular endothelial function and the relationship between IPC, flow-mediated dilation (FMD), and brachial artery diameter (BAD). Methods. Systematic searches were conducted in PubMed, Medline, Cochrane Library, Embase, and Scopus databases from their inception to March 20, 2020. This research included randomized controlled trials (RCTs) with adults, and the values of FMD and BAD were considered as the primary outcomes. Ten studies comprising 292 participants were included in the meta-analysis. Results. Regarding FMD, we observed beneficial effects of IPC on endothelial function (standardized mean difference (SMD): 1.82; 95\% confidence interval (CI): 0.64, 3.01; $p<0.001 ; I^{2}=89.9 \%$ ). However, the available evidence did not indicate that IPC affected BAD (SMD: 0.08; 95\% CI: -0.03 , $\left.0.18 ; p>0.05 ; I^{2}=76.5 \%\right)$. Conclusions. Our meta-analysis indicated a significant effect of IPC on the endothelial function of the blood vessels, affecting FMD but not BAD.

\section{Introduction}

Currently, approximately 120 million people in the US have different forms of cardiovascular disease (CVD), which is considered to be the leading cause of death, morbidity, and disability. In 2016, CVD accounted for about 840,000 deaths in the United States, and the number of CVD deaths increased from 2011 to 2017 by $9.7 \%$ [1]. The loss of function of the endothelium is considered to be an early pathogenic step in the development of atherosclerotic lesions and the subsequent onset of cardiovascular diseases [2]. Therefore, the endothelium has been identified as a tractable physiological target for therapeutic interventions to reduce the risk of CVDs such as coronary heart disease, stroke, or atherosclerosis [3]. Endothelium, which lines the inside of the blood vessels, regulates vascular integrity, reduces thrombosis, decreases vascular tone, improves vascular wall function, and promotes angiogenesis by releasing distinct signaling molecules $[4,5]$. Moreover, the dysfunction of endothelium causes multiple diseases such as pathogenesis and progression of atherosclerosis, cerebrovascular disease, and inflammatory diseases [6]. Thus, the assessment of endothelial function could predict future cardiovascular events and provide an appropriate marker for blood vessels.

Nowadays, some noninvasive techniques have been developed to assess endothelial functions, such as FMD, which represents an endothelium-dependent, primarily nitric oxide- (NO-) mediated dilation of conduit arteries in response to an imposed increase in blood flow and shear stress. Moreover, impaired FMD has been associated with the predisposition to atherosclerosis and CVD and represents an early process in the development of target organ damage and clinical events [7]. In the 1990s, high-frequency ultrasonographic imaging of the brachial artery was developed to assess endothelium-dependent FMD. This technique stimulates the release of nitric oxide, resulting in vasodilation that can be quantitated as an index of vasomotor function [8]. 
IPC, originally proposed by Murry and his group in 1986 , is associated with the ability of endogenous mechanisms to produce strong resistance to ischemic damage shortly after nonlethal mild ischemia or reperfusion treatment [9]. Animal studies [10, 11] have confirmed that IPC reduces ischemia-reperfusion injury in multiple organs. Much time and effort have been devoted to exploring the underlying molecular mechanisms of IPC. The most noteworthy result was that clinical research paid more attention to applying IPC for preventing distal organ damage. IPC was shown to limit the deleterious effects of prolonged ischemia or ischemia/reperfusion (IR), such as complex cardiac surgery, resection of abdominal aortic aneurysm, and kidney transplant operation, particularly in high-risk surgical patients. Many clinical studies have shown that IPC provided significant protection to the cardiac and vascular systems, improving the microcirculation state of blood vessels and maintaining endothelial function. Thus, IPC is expected to be an important therapeutic strategy to alleviate IR injury of vital organs in the future. However, FMD was calculated as a relative percentage change in the baseline $\mathrm{BAD}$ during reactive hyperemia; baseline $\mathrm{BAD}$ was also regarded as an important determinant of FMD [12].

Many clinical studies have analyzed the effects of IPC on endothelial function. Remote IPC before cardiac surgery increased myocardial salvage and protected endothelial function; additionally, it was safer and more economical than other alternatives [13]. Several studies have shown that IPC by transient limb ischemia reduces myocardial IPC injury in patients [13-17]. However, few studies presented protective effects of IPC against endothelial IR injury in patients who had suffered heart failure [18].

This study was conducted to systematically summarize the pieces of evidence for the effects of IPC on endothelial function and conduct a meta-analysis. In this study, two key indicators, FMD and BAD, were evaluated before and after ischemic treatment, and the effects of IPC on endothelial function were systematically examined for the first time in this study. We also evaluated FMD and BAD to further determine the interrelationships between the brachial artery variables and the cardiovascular risk events in a large wellcharacterized population.

\section{Methods}

Our systematic review was conducted according to the guidelines of the Preferred Reporting Items for Systematic Review and Meta-Analyses (Supplementary Table: PRISMAP) [19], and the study protocol was registered with the identification code PROSPERO:CRD42020176093.

2.1. Data Sources and Search Strategy. Five databases (PubMed, Medline, Embase, Cochrane, and Scopus) were used to search for articles from inception until March 20, 2020. Additionally, a manual search of the list of references was performed for the relevant reviews and articles included in the systematic review. A search strategy was developed using the following $\mathrm{MeSH}$ and text keywords: intervention ("ischemic preconditioning" or "remote ischemic preconditioning" or "ischemic") and outcomes ("FMD" or "BAD" or "resting diameter" or "brachial artery flow-mediated dilation" or "vasodilation" or "vascular reactivity" and "endothelial function").

2.2. Literature Selection. Original studies were included if they met the following inclusion criteria: (1) relevant human intervention studies (subjects $\geq 18$ years old), (2) performed IPC, (3) had a control group, and (4) measured endothelial function, including FMD and BAD.

Studies were excluded when (1) they had no information on the intervention or a control group, (2) duplicate publications or substudies of the RCTs were selected, (3) the studies were observational with cross-sectional, case-control, or cohort design, (4) the studies lacked sufficient BAD or FMD information, baseline, or follow-up, and (5) the studies were published in languages other than English.

2.3. Data Extraction. Two independent researchers screened the retrieved articles for eligibility. First, the title and abstract of all the studies were reviewed. Then, the full text of relevant studies was retrieved and assessed to ascertain the suitability of the study for inclusion in the meta-analysis. Any disagreement was discussed and resolved by the third researcher. After data extraction, the following information was recorded in a database: first author's name, publication year, sex, sample size, study design, intervention, duration of the study, and the mean and standard deviation for FMD and BAD in every intervention group and control group. Then, a random-effects meta-analysis was conducted, followed by meta-regression and subgroup analyses to determine whether the effects were modified by health status (i.e., healthy participants versus participants with other diseases), age, gender, ethnicity, and treatment duration.

2.4. Quality Assessment. The quality of the studies was assessed by two independent investigators using the Cochrane Collaboration risk of bias tool and met the following criteria: "random sequence generation, allocation concealment, blinding of participants and personnel, blinding of outcome assessment, incomplete outcome data, selective reporting, and other bias." Based on the recommendations of the Cochrane Handbook, a decision of "yes" indicated a low risk of bias, while "no" indicated a high risk of bias. Labeling an item as "unclear" suggested an unclear or unknown risk of bias $[19,20]$. Any disagreement was discussed and resolved by the third investigator.

2.5. Statistical Analysis. All statistical analyses for our metaanalysis were performed by the open-source statistical software Review Manager (RevMan, Version 5.3.5; The Nordic Cochrane Centre, The Cochrane Collaboration, Copenhagen, Denmark) and Stata version 15 (Stata Corp LLC, Texas, USA). Inverse variance weighting was used to pool the different studies [21]. Potential sources of heterogeneity were investigated by stratified meta-analyses 
according to various study characteristics defined a priori: year, characteristics, different interventions, and duration of intervention. Heterogeneity in the results was quantified by the $I^{2}$ statistic [22]. Sensitivity analyses were performed to assess the robustness of the meta-analysis by removing one study at a time. Publication bias was assessed by visual inspection of the funnel plot [23] and Egger's regression test. The "trim and fill" method by Duval and Tweedie was used to adjust the analysis for the effects of publication bias [24].

\section{Results}

3.1. Study Characteristics. The literature search identified 7,038 articles. Ultimately, ten studies with 12 trials were included in our research. A flow diagram of the process of selecting the studies is shown in Figure 1, and the details of the included studies are presented in Table 1.

In total, 292 participants were listed. The studies included were conducted in America, Europe, and Asia. The intervention period in these studies ranged between one day and eight weeks. The values of FMD and BAD were considered as outcomes.

3.2. Quality Assessment and Potential Bias. The quality score and risk of bias for each study are shown in Figure 2. The outcome of the quality assessment is provided in Table 1 . All studies were randomized [18, 25-33], and four of the studies had additionally conducted before and after randomized trials $[18,33]$. While it was hard to blind researchers and participants to the IPC protocol order, blinding the assessment of outcomes was performed in five studies [26-30]. Distribution concealment and reporting bias were not mentioned in any of the studies, which might have produced certain types of bias.

3.3. Effects of IPC on FMD. Meta-analysis of the 12 sets of independent results showed that IPC improved endothelial function (SMD: 1.824; 95\% CI: 0.64, 3.01; $p<0.05$, shown in Figure 3). Heterogeneity between studies was significant $\left(Q=99.13 ; I^{2}=89.9 \% ; p<0.05\right)$. The results indicated that IPC had a positive effect on the improvement of vascular function. The IPC group increased FMD by 1.82 compared to the FMD in the untreated group. Given that the methods for testing macrovascular and microvascular endotheliumindependent reactivity remained largely unstandardized, meta-regression was only performed on the FMD data. Then, we performed subgroup analysis. The remaining heterogeneity after subgroup meta-analyses (Table 2) showed that age, gender, health status, ethnicity, and treatment duration might be responsible for the substantial amount of heterogeneity among the studies.

3.4. Effects of IPC on BAD. Meta-analysis of the eight sets of independent results showed that IPC did not affect changes in BAD (SMD: 0.08 ; 95\% CI: $-0.03,0.18 ; p=0.148$, shown in Figure 4). Heterogeneity between studies was significant $\left(Q=29.73 ; I^{2}=76.5 \%, p<0.001\right)$, which was due to the variations in the characteristics of the populations (age, gender, or health status), different procedures and methods used (measurements of vascular function and intervention duration), and differences in the study design and quality of research. However, the sources of heterogeneity could not be fully determined since the number of included studies and the sample size of the majority of the studies were relatively small.

3.5. Sensitivity Analyses and Publication Bias. The sensitivity analyses of FMD with the random-effects models are shown in Figure 5. Removing studies individually did not substantially modify the differences in the effect on FMD. Visual inspection of the funnel plot (Figure 6) suggested that, overall, there was no evidence of publication bias, which was also confirmed by Egger's Regression test ( $p=0.813$; Figure 7) and Beeg's test $(p=0.35)$.

\section{Discussion}

Overall, the results of the meta-analysis demonstrated that IPC protected endothelial function and improved FMD. However, it did not show any significant increase in BAD following the application of IPC.

Murry et al. first described the phenomenon of IPC [9]; several studies had demonstrated that IPC could reliably provide myocardial protection $[34,35]$. The breakthrough in the clinical applicability of preconditioning protection came with the discovery by Kharbanda et al. that transient limb ischemia provided cardiovascular protection in humans and animals [36, 37]. Besides, IPC used as an adjunct to primary percutaneous coronary intervention in patients with STelevation myocardial infarction improved long-term clinical outcomes [17].

Endothelial dysfunction is involved in the development of atherosclerosis, which precedes asymptomatic structural vascular alterations, as well as clinical manifestations of CVD. Endothelial function can be assessed noninvasively using the FMD technique. Therefore, we can improve FMD through IPC and indirectly reduce the risk of related diseases. As an emerging detection indicator, FMD is closely related to the occurrence and development of many diseases. Moreover, brachial artery FMD has been used independently to predict long-term adverse CV events in healthy subjects with no apparent heart disease in addition to being used for assessing some traditional risk factors [38]. Besides, Perri et al. [39] found that AF patients with low FMD were associated with an increased risk of CVE (cardiovascular events), suggesting that impaired artery dilation predisposes to atherosclerotic complications. Additionally, some researchers suggested that the combination of FMD and nitroglycerine-induced vasodilation measurements could more accurately predict cardiovascular events than by measuring vasodilation with nitroglycerine only [40]. One study on systemic lupus erythematosus showed that the accumulation of damage in patients was associated with a progressive loss of FMD, with preserved endothelium-independent vasodilation [41]. Han et al. [42] found that low 


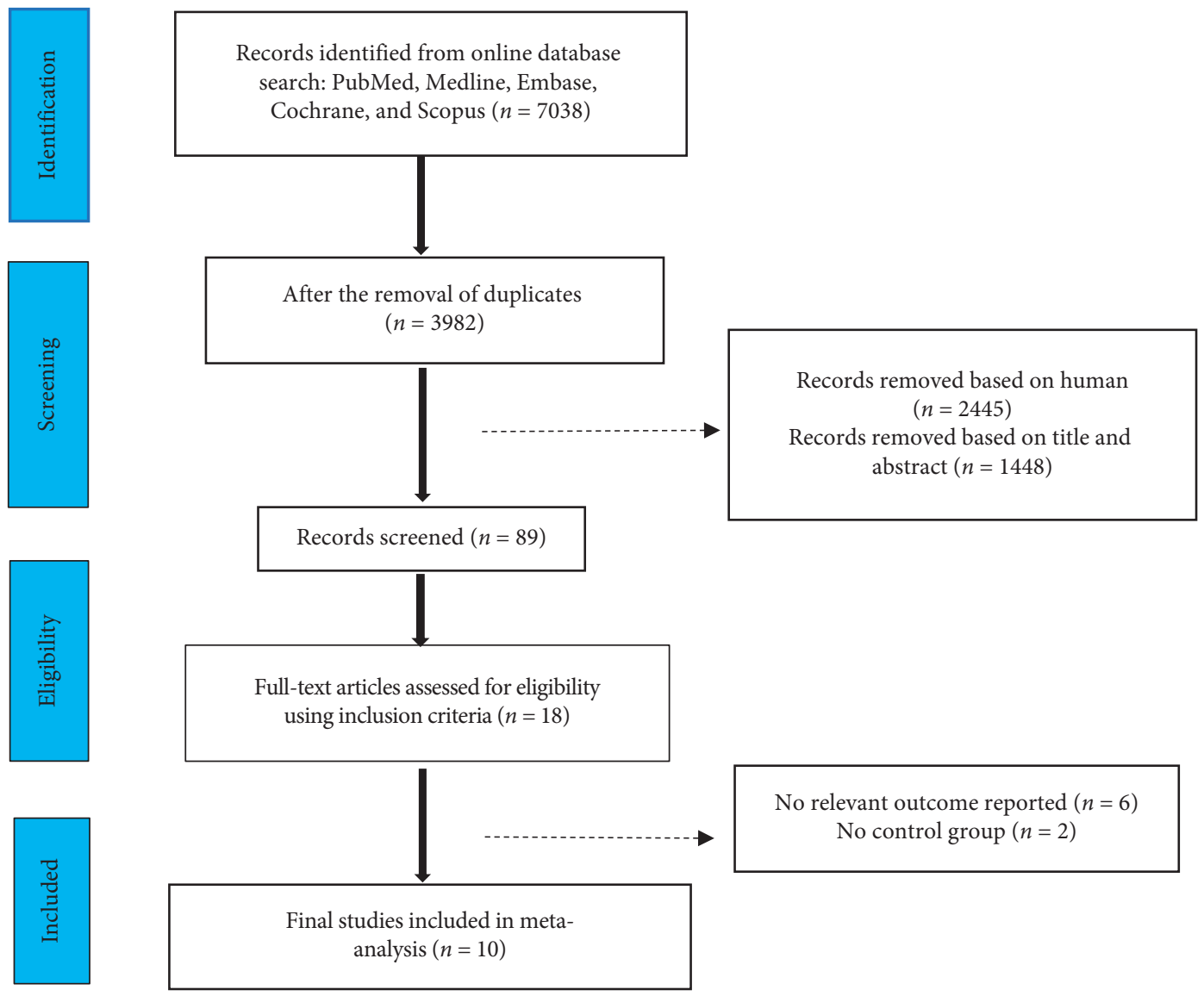

FIGURE 1: Flowchart showing the literature searched and reviewed for the selection of the studies.

TABLE 1: Details of the included studies.

\begin{tabular}{|c|c|c|c|c|c|c|c|c|}
\hline Author & Publication year & Male & Country & $\begin{array}{c}\text { Sample size (control/ } \\
\text { intervention) }\end{array}$ & Age & Intervention & Duration & $\begin{array}{l}\text { Presented } \\
\text { data }\end{array}$ \\
\hline Jones et al. [25] & 2014 & 16 & England & $8 / 8$ & 24 & IPC or not & 8 weeks & FMD, BAD \\
\hline Liuni et al. [26] & 2010 & 20 & America & $10 / 10$ & $18-33$ & IPC or not & 5 days & FMD, BAD \\
\hline Liuni et al. [27] & 2011 & 18 & America & $9 / 9$ & $18-29$ & IPC or not & 20 days & FMD, BAD \\
\hline Seeger et al. [18] & 2014 & 15 & The Netherlands & $15 / 15$ & 67 & IPC or not & 7 days & FMD, BAD \\
\hline Seeger et al.* [18] & 2014 & 15 & The Netherlands & $15 / 15$ & 65 & IPC or not & 7 days & FMD, BAD \\
\hline Verouhis et al. [28] & 2019 & 4 & The Swedish & $4 / 4$ & 30.5 & IPC or not & 6 days & FMD, BAD \\
\hline Bailey et al. [29] & 2012 & 11 & England & $11 / 11$ & 25 & IPC or not & Immediate & $\mathrm{FMD}, \mathrm{BAD}$ \\
\hline Luca et al. [30] & 2013 & 15 & Canada & $15 / 15$ & $20-31$ & IPC or not & 1 day & FMD, BAD \\
\hline Liang et al. [31] & 2015 & 20 & China & $20 / 20$ & 64 & IPC or not & 20 days & FMD \\
\hline Manchurov et al. [32] & 2014 & 26 & Russia & $25 / 23$ & 62 & IPC or not & 7 days & FMD \\
\hline Munckhof et al. [33] & 2013 & 15 & The Netherlands & $15 / 15$ & 72 & IPC or not & 7 days & FMD \\
\hline Munckhof et al.* [33] & 2013 & 15 & The Netherlands & $15 / 15$ & 22 & IPC or not & 7 days & FMD \\
\hline
\end{tabular}

${ }^{*}$ From the same article.

baseline FMD in hyperuricemia patients was associated with a significantly increased risk of incident hypertension and that FMD could be used as one of the predictive factors of the risk of diseases.

There are several mechanisms through which FMD could improve endothelial function and might account for the beneficial effects observed in this study. First, cardiovascular protection provided by the early phase of IPC is mediated by the stimulation of receptors linked to protein kinase C (PKC) activation by adenosine, bradykinin, NO, and free radicals [43-45]. Recently, Kharbanda et al. [36] reported that IPC might help to reduce endothelial injury during ischemic reperfusion in humans. Subsequent studies in humans have confirmed that IPC decreased inflammatory 


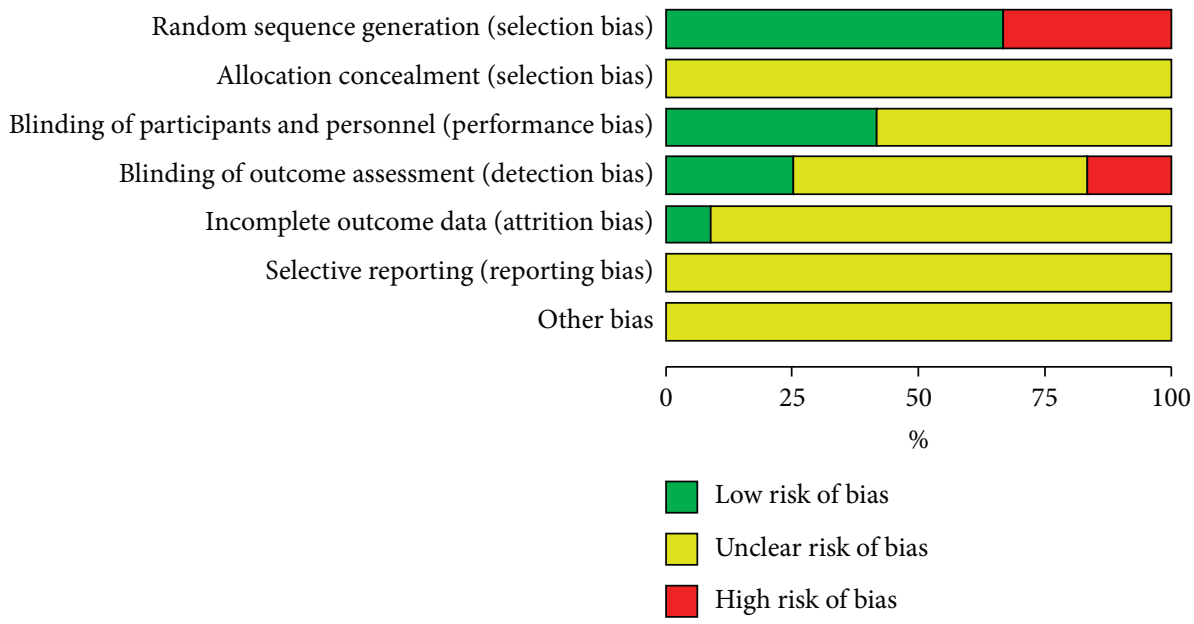

FIGURE 2: Summary of the risk of bias.

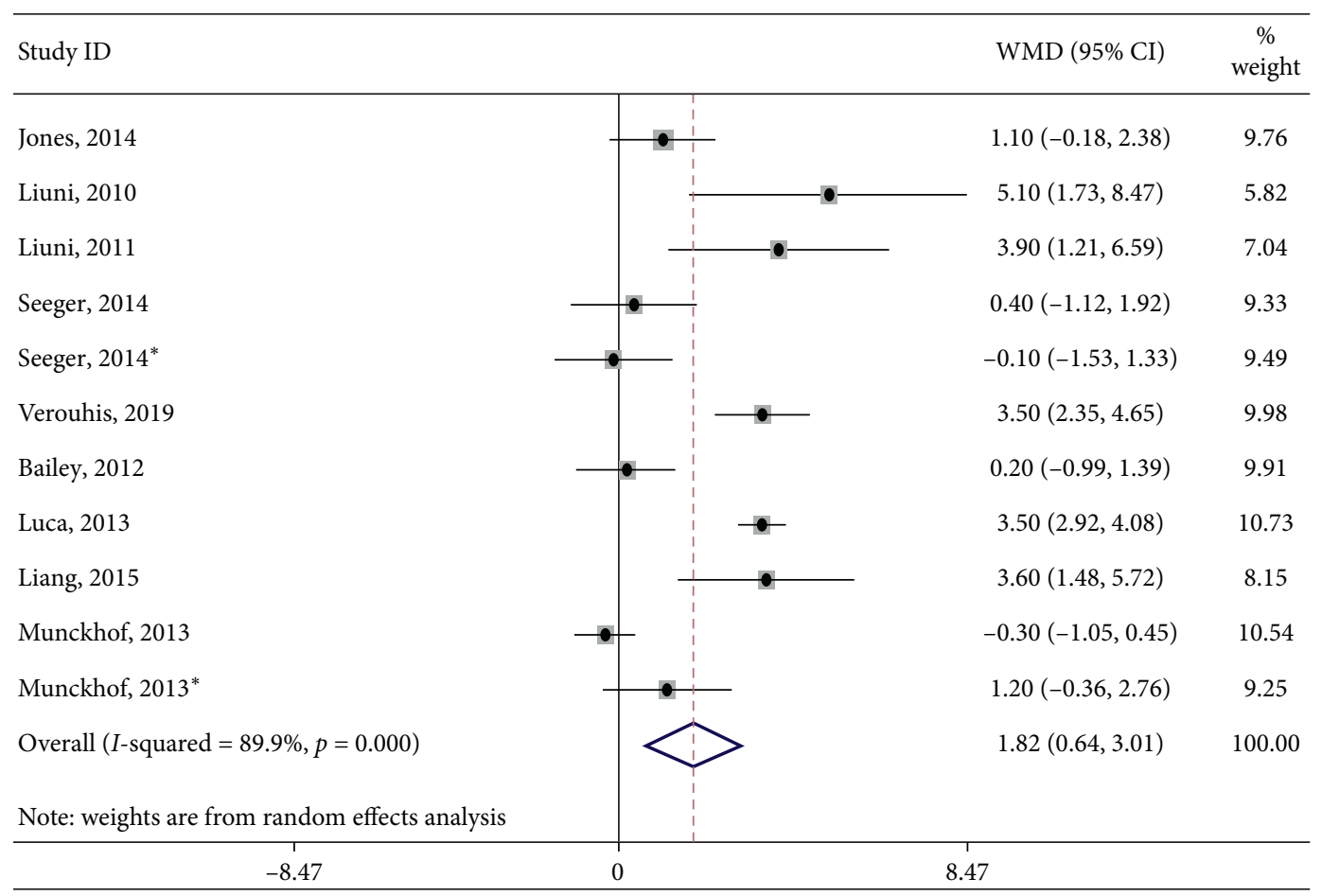

FIGURE 3: Forest plot showing the overall effect of IPC on flow-mediated dilation in adults (expressed as a percentage change). Data are shown as the percentage differences in means. Horizontal lines denote $95 \% \mathrm{CI}$. The size of the boxes is proportionally scaled to the effect size for each study.

reaction and improved endothelial function by these humoral mediators [46-48]. One possible mechanism by which repetition of IPC augments endothelial function is by stepping up the vascular shear stress resulting from increased blood flow. Acute or chronic increase in the shear stress stimulates the release of NO in the blood vessels [49]. Additionally, a steady increase in shear stress has been shown to cause functional and histological alterations of the vascular endothelium, resulting in enhanced vascular structure and function [50]. This beneficial change in the endothelium after the repetition of IPC also might contribute to the augmented forearm vascular response to ACh (acetylcholine) and the ACh-stimulated NO release. On the other hand, preconditioning stimulus did not directly alter the endothelial function but avoided endothelial dysfunction in both the conduit and resistance vessels in response to IR.

In conclusion, our findings indicated that IPC augmented the endothelial function through an increase in FMD. It is important to select an appropriate intervention that is effective in improving or augmenting endothelial 
TABLE 2: Subgroup analyses for the effects of FMD on the markers of endothelial function.

\begin{tabular}{|c|c|c|c|c|c|c|c|c|}
\hline \multirow{2}{*}{ Trial characteristic } & \multicolumn{2}{|c|}{$\begin{array}{l}\text { Meta-regression } \\
\text { analysis }\end{array}$} & \multicolumn{6}{|c|}{ Subgroup analysis } \\
\hline & $P$ value & $95 \% \mathrm{CI}$ & Stratification variable & Number of effect sizes & $\begin{array}{l}\text { Pooled } \\
\text { WMD }\end{array}$ & $95 \% \mathrm{CI}$ & $\begin{array}{l}P \text { value within } \\
\text { subgroups }\end{array}$ & $\begin{array}{l}I^{2} \\
(\%)\end{array}$ \\
\hline \multirow{2}{*}{ Age } & \multirow{2}{*}{0.16} & \multirow{2}{*}{$(-5.29,1.14)$} & $\leq 35 y$ & 7 & 2.41 & $(1.15,3.68)$ & $<0.001$ & 84.4 \\
\hline & & & $\geq 60 y$ & 5 & 0.66 & $(-0.70,2.01)$ & 0.008 & 74.5 \\
\hline \multirow{2}{*}{ Gender } & \multirow{2}{*}{0.42} & \multirow{2}{*}{$(-3.70,1.82)$} & $>50 \%$ male & 10 & 2.20 & $(0.83,3.56)$ & $<0.001$ & 90.8 \\
\hline & & & $\leq 50 \%$ male & 2 & 0.28 & $(-0.66,1.21)$ & 0.839 & 0 \\
\hline \multirow{2}{*}{ Health status } & \multirow{2}{*}{0.86} & \multirow{2}{*}{$(-4.25,4.90)$} & Asymptomatic & 9 & 1.82 & $(0.47,3.16)$ & $<0.001$ & 91.4 \\
\hline & & & Diseased & 3 & 1.91 & $(-1.22,5.04)$ & 0.016 & 82.7 \\
\hline \multirow{3}{*}{ Ethnicity } & \multirow{3}{*}{0.05} & \multirow{3}{*}{$(-0.45,3.62)$} & American & 3 & 3.56 & $(3.00,4.12)$ & 0.64 & 0 \\
\hline & & & European & 8 & 0.85 & $(-0.21,1.92)$ & $<0.001$ & 81.5 \\
\hline & & & Asian & 1 & 3.60 & $(1.48,5.72)$ & & \\
\hline \multirow{3}{*}{ Treatment duration } & \multirow{3}{*}{0.68} & \multirow{3}{*}{$(-1.99,1.40)$} & $5 \mathrm{~min}$ & 2 & 0.14 & $(-0.91,1.18)$ & 0.64 & 0 \\
\hline & & & $15 \mathrm{~min}$ & 3 & 3.56 & $(3.00,4.12)$ & 0.64 & 0 \\
\hline & & & $20 \mathrm{~min}$ & 7 & 1.45 & $(0.08,2.83)$ & $<0.001$ & 86.6 \\
\hline
\end{tabular}

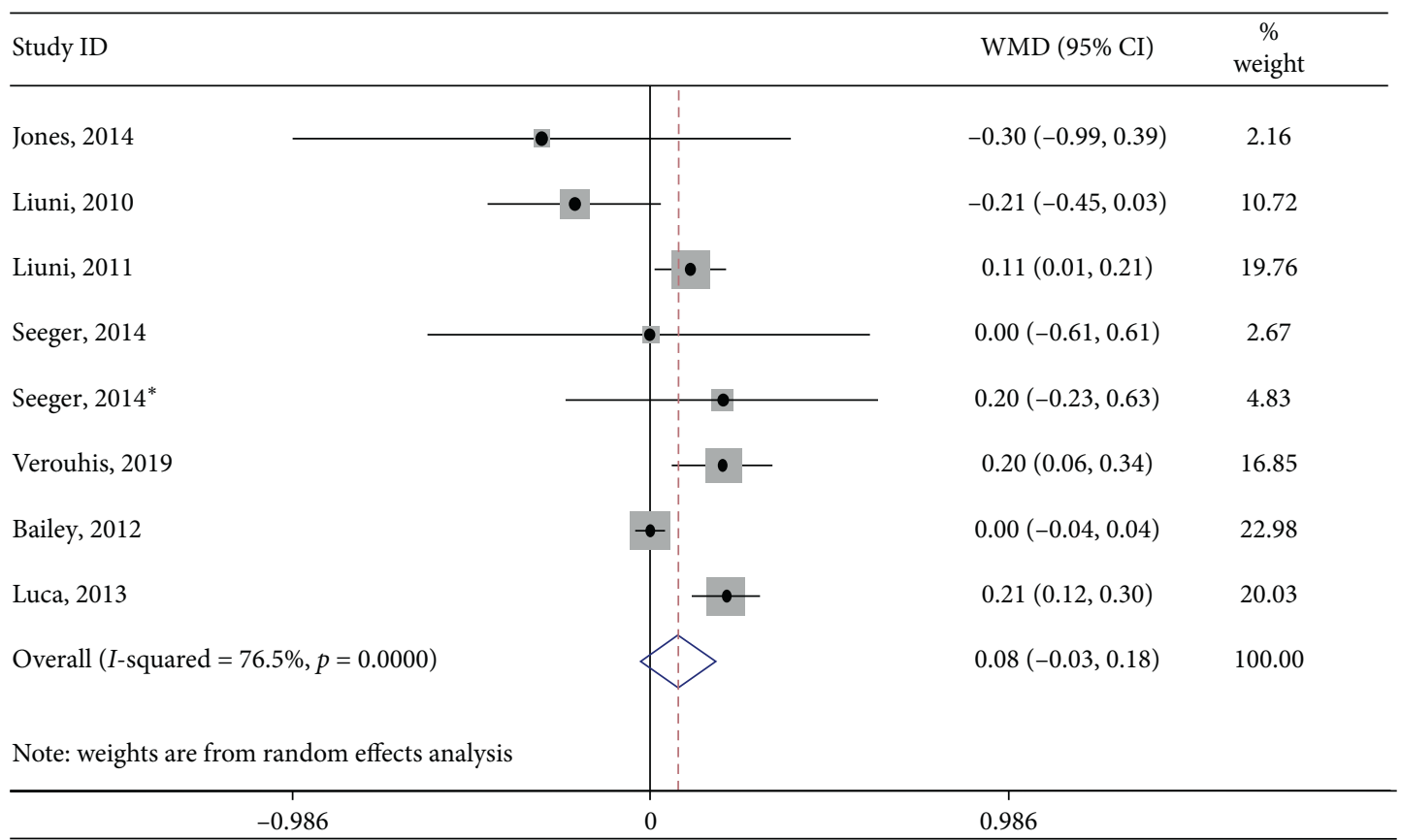

FIGURE 4: Forest plot showing the overall effect of IPC on BAD. Horizontal lines denote $95 \%$ CI. The size of the boxes is proportionally scaled to the effect size for each study.

function. IPC has the potential for improving endothelial function as a novel method for predicting and preventing cardiovascular diseases associated with endothelial dysfunction. Besides, the idea of providing significant myocardial protection with transient limb ischemia is highly attractive to clinicians because it only requires a blood pressure cuff [16]. As a simple, safe, and feasible therapeutic technique, which can easily be applied in the preoperative setting to patients with acute cardiac events, IPC may have the potential to reduce mortality. Although IPC is a part of the most powerful and reproducible phenomenon in cardioprotection, it has not been readily translated into routine clinical use because of methodological hurdles and limitations. Overall, our findings need to be demonstrated in a larger multicenter trial before IPC can be implemented extensively as adjunctive therapy in clinical settings [17].

\section{Limitations}

The overall quality of the studies included was the modest. The majority of the investigations did not allow blind participants to the intervention arm, and no study reported methods of allocation concealment. Additionally, given the small number of studies included in this review, our analysis might have been underpowered to detect differences in the effectiveness of interventions based on health status, type of 


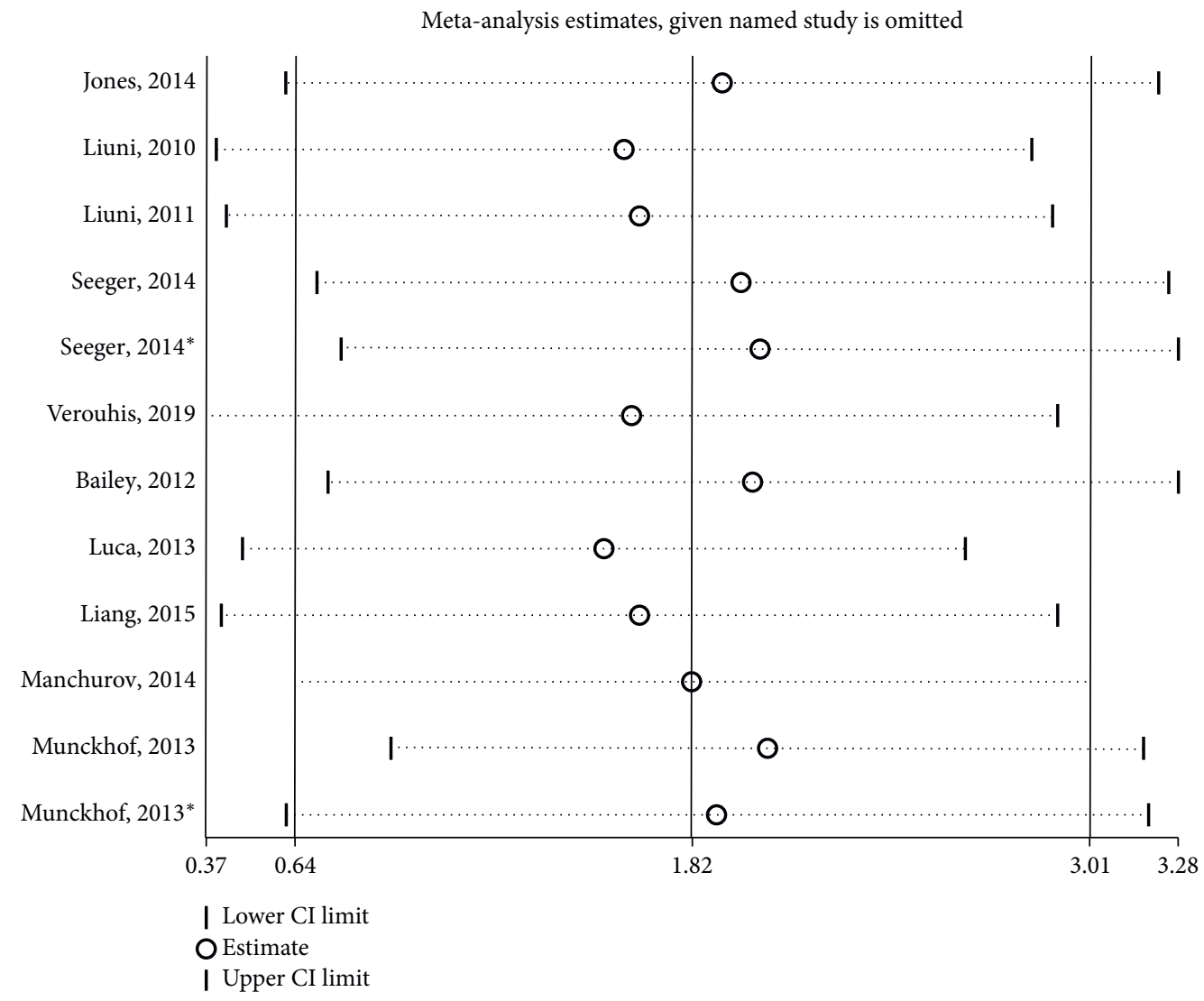

FIgURE 5: Sensitivity analyses of FMD.

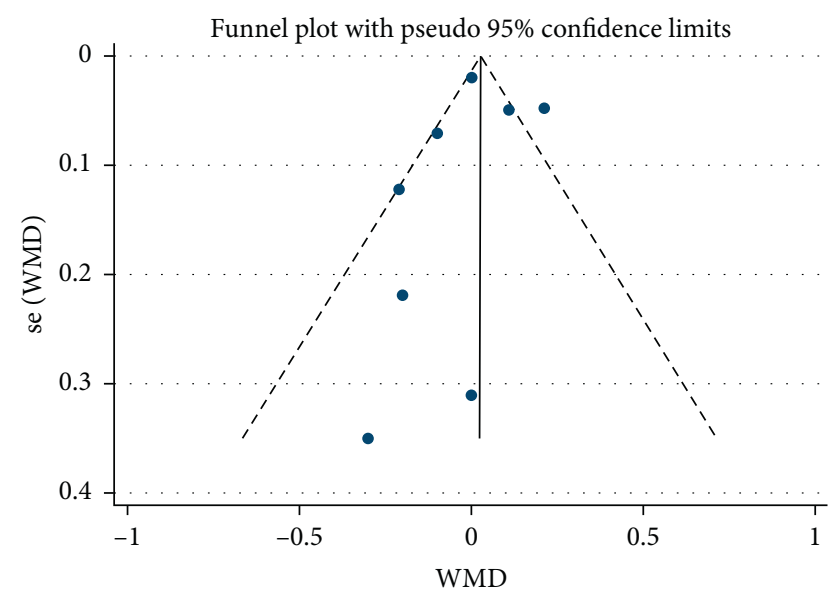

FIGURE 6: Funnel plot used to evaluate publication bias.

measurement, and study design. Differences in the effect size among the included studies could have been affected by the coexistence of traditional CVD risk factors (e.g., hypertension, smoking habit, diabetes mellitus, obesity, and hyperlipidemia) and the concomitant treatments. Further investigation is needed to establish the applicability and safety of IPC in clinical populations. The consideration of the other factors related to changes in cardiovascular risk is also warranted.

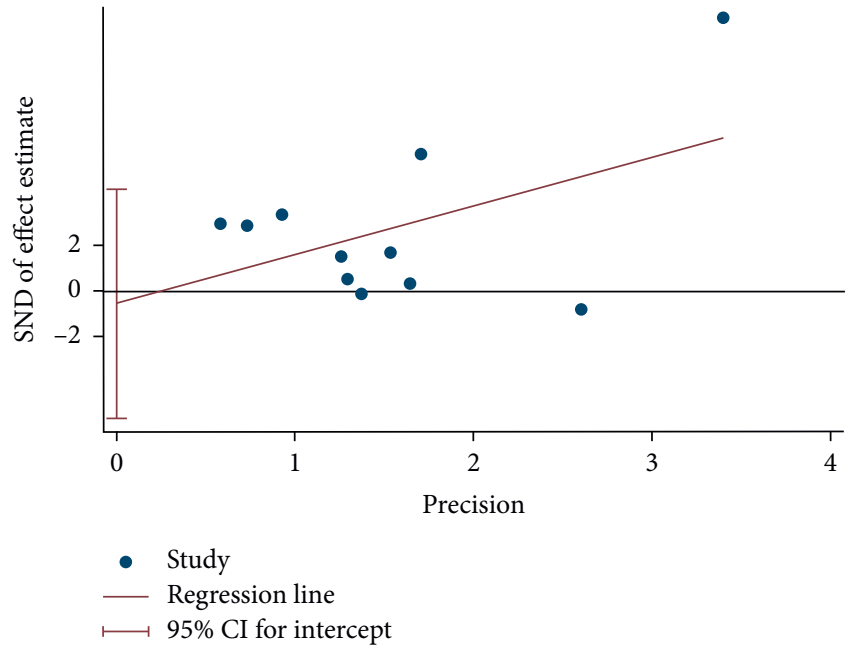

FIgURE 7: Egger's test used to evaluate publication bias.

\section{Conflicts of Interest}

The authors declare that they have no conflicts of interest.

\section{Authors' Contributions}

Xufang Gu and Zhichao Liu contributed equally to the work. $\mathrm{Gu}$ is the guarantor. Liu drafted the manuscript. Wang, Gao, 
and Chen collected and assessed data. Wang and Gao collaborated with Liu and revised the study. $\mathrm{Li}$ and $\mathrm{Gu}$ developed search strategy. Two independent researchers (Liu and Chen) assessed the quality of all studies; if there is a difference, a third researcher $(\mathrm{Li})$ was invited to solve. All authors contributed to the development of the selection criteria, the risk of a bias assessment strategy, and data extraction criteria. They also read, provided feedback, and approved the final draft of the manuscript.

\section{Acknowledgments}

This research was funded by the National Natural Science Foundation of China (81873149).

\section{Supplementary Materials}

Supplemental Table: PRISMA-P. (Supplementary Materials)

\section{References}

[1] V. L. Roger, S. Sidney, A. L. Fairchild et al., "Recommendations for cardiovascular health and disease surveillance for 2030 and beyond: a policy statement from the American Heart Association," Circulation, vol. 141, no. 9, pp. e104-e119, 2020.

[2] P. M. Vanhoutte, H. Shimokawa, M. Feletou, and E. H. C. Tang, "Endothelial dysfunction and vascular disease a 30th anniversary update," Acta Physiologica, vol. 219, no. 1, pp. 22-96, 2017.

[3] M. Siervo, F. Scialò, O. M. Shannon, B. C. M. Stephan, and A. W. Ashor, "Does dietary nitrate say NO to cardiovascular ageing? Current evidence and implications for research," Proceedings of the Nutrition Society, vol. 77, no. 2, pp. 112-123, 2018.

[4] S. Godo and H. Shimokawa, "Endothelial functions," Arteriosclerosis, Thrombosis, and Vascular Biology, vol. 37, no. 9, pp. e108-e114, 2017.

[5] J. Lara, A. W. Ashor, C. Oggioni, A. Ahluwalia, J. C. Mathers, and M. Siervo, "Effects of inorganic nitrate and beetroot supplementation on endothelial function: a systematic review and meta-analysis," European Journal of Nutrition, vol. 55, no. 2, pp. 451-459, 2016.

[6] D. Konukoglu and H. Uzun, "Endothelial dysfunction and hypertension," Advances in Experimental Medicine and Biology, vol. 956, pp. 511-540, 2017.

[7] D. H. J. Thijssen, R. M. Bruno, A. C. C. M. van Mil et al., "Expert consensus and evidence-based recommendations for the assessment of flow-mediated dilation in humans," $E u$ ropean Heart Journal, vol. 40, no. 30, pp. 2534-2547, 2019.

[8] D. S. Celermajer, K. E. Sorensen, V. M. Gooch et al., "Noninvasive detection of endothelial dysfunction in children and adults at risk of atherosclerosis," The Lancet, vol. 340, no. 8828, pp. 1111-1115, 1992.

[9] C. E. Murry, R. B. Jennings, and K. A. Reimer, "Preconditioning with ischemia: a delay of lethal cell injury in ischemic myocardium," Circulation, vol. 74, no. 5, pp. 1124-1136, 1986.

[10] D. C. Hess, R. A. Blauenfeldt, G. Andersen et al., "Remote ischaemic conditioning-a new paradigm of self-protection in the brain," Nature Reviews Neurology, vol. 11, no. 12, pp. 698-710, 2015.

[11] S. Mastitskaya, M. Basalay, P. S. Hosford, A. G. Ramage, A. Gourine, and A. V. Gourine, "Identifying the source of a humoral factor of remote (Pre)conditioning cardioprotection," PLoS One, vol. 11, no. 2, Article ID e0150108, 2016.

[12] T. Maruhashi, Y. Iwamoto, M. Kajikawa et al., "Interrelationships among flow-mediated vasodilation, nitroglycerineinduced vasodilation, baseline brachial artery diameter, hyperemic shear stress, and cardiovascular risk factors," Journal of the American Heart Association, vol. 7, no. 1, 2017.

[13] R. M. Ahmed, E.-H. A. Mohamed, M. Ashraf et al., "Effect of remote ischemic preconditioning on serum troponin T level following elective percutaneous coronary intervention," Catheterization and Cardiovascular Interventions, vol. 82, no. 5, pp. E647-E653, 2013.

[14] H. E. Bøtker, R. Kharbanda, M. R. Schmidt et al., "Remote ischaemic conditioning before hospital admission, as a complement to angioplasty, and effect on myocardial salvage in patients with acute myocardial infarction: a randomised trial," Lancet (London, England), vol. 375, no. 9716, pp. 727-734, 2010.

[15] D. Brevoord, P. Kranke, M. Kuijpers, N. Weber, M. Hollmann, and B. Preckel, "Remote ischemic conditioning to protect against ischemia-reperfusion injury: a systematic review and meta-analysis," PLoS One, vol. 7, no. 7, Article ID e42179, 2012.

[16] S. J. Luo, Y. J. Zhou, D. M. Shi, H. L. Ge, J. L. Wang, and R. F. Liu, "Remote ischemic preconditioning reduces myocardial injury in patients undergoing coronary stent implantation," Canadian Journal of Cardiology, vol. 29, no. 9, pp. 1084-1089, 2013.

[17] A. D. Sloth, M. R. Schmidt, K. Munk et al., "Improved longterm clinical outcomes in patients with ST-elevation myocardial infarction undergoing remote ischaemic conditioning as an adjunct to primary percutaneous coronary intervention," European Heart Journal, vol. 35, no. 3, pp. 168-175, 2014.

[18] J. P. Seeger, N. M. Benda, N. P. Riksen et al., "Heart failure is associated with exaggerated endothelial ischaemia-reperfusion injury and attenuated effect of ischaemic preconditioning," European Journal of Preventive Cardiology, vol. 23, no. 1, pp. 33-40, 2016.

[19] A. Liberati, D. G. Altman, J. Tetzlaff et al., “The PRISMA statement for reporting systematic reviews and meta-analyses of studies that evaluate healthcare interventions: explanation and elaboration," Bmj, vol. 339, p. b2700, 2009.

[20] M. Cumpston, T. Li, M. J. Page et al., "Updated guidance for trusted systematic reviews: a new edition of the Cochrane Handbook for Systematic Reviews of Interventions," The Cochrane Database of Systematic Reviews, vol. 10, Article ID Ed000142, 2019.

[21] F. O. Curtin, D. Elbourne, and D. G. Altman, "Meta-analysis combining parallel and cross-over clinical trials. III: the issue of carry-over," Statistics in Medicine, vol. 21, no. 15, pp. 2161-2173, 2002.

[22] W. G. Melsen, M. C. J. Bootsma, M. M. Rovers, and M. J. M. Bonten, "The effects of clinical and statistical heterogeneity on the predictive values of results from metaanalyses," Clinical Microbiology and Infection, vol. 20, no. 2, pp. 123-129, 2014.

[23] J. A. C. Sterne and M. Egger, "Funnel plots for detecting bias in meta-analysis: guidelines on choice of axis," Journal of Clinical Epidemiology, vol. 54, no. 10, pp. 1046-1055, 2001.

[24] S. Duval and R. Tweedie, "Trim and fill: a simple funnel-plotbased method of testing and adjusting for publication bias in meta-analysis," Biometrics, vol. 56, no. 2, pp. 455-463, 2000. 
[25] H. Jones, J. Nyakayiru, T. G. Bailey et al., "Impact of eight weeks of repeated ischaemic preconditioning on brachial artery and cutaneous microcirculatory function in healthy males," European Journal of Preventive Cardiology, vol. 22, no. 8, pp. 1083-1087, 2015.

[26] A. Liuni, M. C. Luca, T. Gori, and J. D. Parker, "Rosuvastatin prevents conduit artery endothelial dysfunction induced by ischemia and reperfusion by a cyclooxygenase-2-dependent mechanism," Journal of the American College of Cardiology, vol. 55, no. 10, pp. 1002-1006, 2010.

[27] A. Liuni, M. C. Luca, T. Gori, and J. D. Parker, "Loss of the preconditioning effect of rosuvastatin during sustained therapy: a human in vivo study," American Journal of Physiology-Heart and Circulatory Physiology, vol. 302, no. 1, pp. H153-H158, 2012.

[28] D. Verouhis, N. Saleh, M. Settergren, P. Sörensson, A. Gourine, and J. Pernow, "Remote ischemic conditioning protects against endothelial ischemia-reperfusion injury via a glucagon-like peptide-1 receptor-mediated mechanism in humans," International Journal of Cardiology, vol. 274, pp. 40-44, 2019.

[29] T. G. Bailey, G. K. Birk, N. T. Cable et al., "Remote ischemic preconditioning prevents reduction in brachial artery flowmediated dilation after strenuous exercise," American Journal of Physiology-Heart and Circulatory Physiology, vol. 303, no. 5, pp. H533-H538, 2012.

[30] M. C. Luca, A. Liuni, K. McLaughlin, T. Gori, and J. D. Parker, "Daily ischemic preconditioning provides sustained protection from ischemia-reperfusion induced endothelial dysfunction: a human study," Journal of the American Heart Association, vol. 2, no. 1, Article ID e000075, 2013.

[31] Y. Liang, Y. P. Li, F. He, X. Q. Liu, and J. Y. Zhang, "Longterm, regular remote ischemic preconditioning improves endothelial function in patients with coronary heart disease," Brazilian Journal of Medical and Biological Research, vol. 48, no. 6, pp. 568-576, 2015.

[32] V. N. M. Manchurov, N. B. R. Ryazankina, R. Y. R. Reztsov, D. V. S. Skrypnik, E. Y. V. Vasilieva, and A. V. S. Shpektor, "Effect of remote ischemic preconditioning and postconditioning on blood flow in the infarct-related artery and endothelial function in patients with ST-elevation myocardial infarction,” Kardiologiia, vol. 1_2016, no. 1, pp. 6-11, 2016.

[33] I. van den Munckhof, N. Riksen, J. P. H. Seeger et al., "Aging attenuates the protective effect of ischemic preconditioning against endothelial ischemia-reperfusion injury in humans," American Journal of Physiology-Heart and Circulatory Physiology, vol. 304, no. 12, pp. H1727-H1732, 2013.

[34] A. Eisen, E. Z. Fisman, M. Rubenfire et al., "Ischemic preconditioning: nearly two decades of research. A comprehensive review," Atherosclerosis, vol. 172, no. 2, pp. 201-210, 2004.

[35] W. R. Davies, A. J. Brown, W. Watson et al., "Remote ischemic preconditioning improves outcome at 6 years after elective percutaneous coronary intervention: the CRISP stent trial long-term follow-up," Circulation: Cardiovascular Interventions, vol. 6, no. 3, pp. 246-251, 2013.

[36] R. K. Kharbanda, U. M. Mortensen, P. A. White et al., "Transient limb ischemia induces remote ischemic preconditioning in vivo," Circulation, vol. 106, no. 23, pp. 2881-2883, 2002.

[37] C. Piot, P. Croisille, P. Staat et al., "Effect of cyclosporine on reperfusion injury in acute myocardial infarction," New England Journal of Medicine, vol. 359, no. 5, pp. 473-481, 2008.
[38] M. Shechter, A. Shechter, N. Koren-Morag, M. S. Feinberg, and L. Hiersch, "Usefulness of brachial artery flow-mediated dilation to predict long-term cardiovascular events in subjects without heart disease," The American Journal of Cardiology, vol. 113, no. 1, pp. 162-167, 2014.

[39] L. Perri, D. Pastori, P. Pignatelli, F. Violi, and L. Loffredo, "Flow-mediated dilation is associated with cardiovascular events in non-valvular atrial fibrillation patients," International Journal of Cardiology, vol. 179, pp. 139-143, 2015.

[40] M. Kajikawa, T. Maruhashi, E. Hida et al., "Combination of flow-mediated vasodilation and nitroglycerine-induced vasodilation is more effective for prediction of cardiovascular events," Hypertension, vol. 67, no. 5, pp. 1045-1052, 2016.

[41] L. Ghiadoni, C. Tani, R. M. Bruno et al., "Prognostic value of flow mediated dilation in patients with systemic lupus erythematosus: a pilot prospective cohort study," Atherosclerosis, vol. 236, no. 2, pp. 381-384, 2014.

[42] C. Han, Z. Xian, Y. Zou et al., "Flow-mediated dilation can be used to predict incident hypertension in patients with hyperuricemia," Archives of Medical Science, vol. 15, no. 2, pp. 343-349, 2019.

[43] A. Nakano, M. V. Cohen, and J. M. Downey, "Ischemic preconditioning: from basic mechanisms to clinical applications," Pharmacology \& Therapeutics, vol. 86, no. 3, pp. 263-275, 2000.

[44] W. Z. Wang, S. Z. Guo, G. L. Anderson, and F. N. Miller, "The initiating factors of late preconditioning in skeletal muscle," Journal of Surgical Research, vol. 99, no. 1, pp. 92-99, 2001.

[45] W. Z. Wang, L. L. Stepheson, G. L. Anderson, F. N. Miller, K. T. Khiabani, and W. A. Zamboni, "Role of PKC in the late phase of microvascular protection induced by preconditioning," Journal of Surgical Research, vol. 106, no. 1, pp. 166-172, 2002.

[46] M. M. H. Cheung, R. K. Kharbanda, I. E. Konstantinov et al., "Randomized controlled trial of the effects of remote ischemic preconditioning on children undergoing cardiac surgery: first clinical application in humans," Journal of the American College of Cardiology, vol. 47, no. 11, pp. 2277-2282, 2006.

[47] D. W. Harkin, A. A. B. Barros D’Sa, K. McCallion, M. Hoper, and F. C. Campbell, "Ischemic preconditioning before lower limb ischemia-reperfusion protects against acute lung injury," Journal of Vascular Surgery, vol. 35, no. 6, pp. 1264-1273, 2002.

[48] M. Kimura, K. Ueda, C. Goto et al., "Repetition of ischemic preconditioning augments endothelium-dependent vasodilation in humans: role of endothelium-derived nitric oxide and endothelial progenitor cells," Arteriosclerosis, Thrombosis, and Vascular Biology, vol. 27, no. 6, pp. 1403-1410, 2007.

[49] Y. Kawai, Y. Yokoyama, M. Kaidoh, and T. Ohhashi, "Shear stress-induced ATP-mediated endothelial constitutive nitric oxide synthase expression in human lymphatic endothelial cells," American Journal of Physiology-Cell Physiology, vol. 298, no. 3, pp. C647-C655, 2010.

[50] J. Niebauer and J. P. Cooke, "Cardiovascular effects of exercise: role of endothelial shear stress," Journal of the American College of Cardiology, vol. 28, no. 7, pp. 1652-1660, 1996. 